\title{
Segregating partition problem in competition-diffusion systems
}

\author{
SHIN-ICHIRO EI \\ Graduate School of Integrated Science, Yokohama City University, 22-2 Seto, Kanazawa-Ku, \\ Yokohama 236-0027, Japan \\ RYO IKOTA \\ Graduate School of Mathematical Sciences, University of Tokyo, 3-8-1 Komaba, Meguro-Ku, \\ Tokyo 153-8914, Japan \\ AND \\ MASAYASU MimuRA \\ Department of Mathematics, Hiroshima University, 1-3-1 Kagamiyama, Higashi-Hiroshima \\ 739-8526, Japan
}

[Received 12 November 1998 and in revised form 21 January 1999]

\begin{abstract}
We consider a reaction-diffusion system to describe the interaction of three competing species which move by diffusion in $\mathbf{R}^{2}$, under the situation where all of the diffusion rates are small and all of the inter-specific competition rates are large. The resulting system possesses three locally stable spatially constant equilibria, each of which implies that only one of the competing species survive and the other two are extinct. Since the diffusion rates are small, internal layer regions appear as sharp interfaces with triple junctions, which generally divide the whole plane into three different regions occupied by only one of the species. The dynamics of interfaces as well as triple junctions are numerically studied. More specifically, assuming that three competing species are almost equal in competitive strength, we derive an angle condition between any neighboring interfaces at triple junctions by formal asymptotic analysis.

Furthermore, for more general cases, we numerically study the dynamics of segregating patterns of three competing species from interfacial view points.
\end{abstract}

\section{Introduction}

Understanding of spatial and/or temporal behaviors of ecologically interacting species is a central problem in population ecology. As for competitive interactions of species, problems of coexistence and exclusion have been theoretically investigated by using different types of mathematical models. Specifically, a variety of reaction-diffusion (RD) equations have been proposed to study spatial segregation of competing species. Quite recently, the methods which are called 'spatial segregation limits' have been successfully developed in mathematical communities [3,4]. These enable us to derive evolutional equations describing the boundaries of spatially segregating patterns of competing species. In some situations, the derived equations are described by new types of free boundary problems.

As a well known model, we are concerned with a RD system of Gause-Lotka-Volterra type [6]. Let $u_{i}(t, \mathbf{x})$ be the population density of the $i$ th species $U_{i}(i=1,2, \ldots, n)$ at time $t>0$ and the position $\mathbf{x} \in \Omega$, where $\Omega$ is a bounded domain in $\mathbf{R}^{2}$. The resulting system for $u_{i}(i=1,2, \ldots, n)$ 
is given by

$$
\frac{\partial u_{i}}{\partial t}=D_{i} \Delta u_{i}+f_{i}\left(u_{1}, u_{2}, \ldots, u_{n}\right) \quad(i=1,2, \ldots, n), \quad t>0, \mathbf{x} \in \Omega .
$$

Here $D_{i}$ is the diffusion rate of $u_{i}$ and $f_{i}=\left(r_{i}-\sum_{i, j}^{n} a_{i j} u_{j}\right) u_{i}$ where $r_{i}$ is the intrinsic growth rate, $a_{i i}$ and $a_{i j}$ are the intraspecific and the interspecfic competition rates, respectively $(i, j=$ $1,2, \ldots, n)$. All of the rates are assumed to be positive constants. We impose the zero-flux boundary conditions

$$
\frac{\partial u_{i}}{\partial v}=0(i=1,2, \ldots, n), \quad t>0, \mathbf{x} \in \partial \Omega,
$$

where $v$ is the outward normal unit vector on $\partial \Omega$. The initial conditions are

$$
u_{i}(0, \mathbf{x})=u_{0 i}(\mathbf{x}) \geqslant 0 \quad(i=1,2, \ldots, n), \quad \mathbf{x} \in \Omega
$$

The simplest system of (1.1) is the case with $n=2$, that is,

$$
\left\{\begin{array}{l}
\frac{\partial u_{1}}{\partial t}=D_{1} \Delta u_{1}+\left(r_{1}-a_{1} u_{1}-b_{1} u_{2}\right) u_{1}, \\
\frac{\partial u_{2}}{\partial t}=D_{2} \Delta u_{2}+\left(r_{2}-b_{2} u_{1}-a_{2} u_{2}\right) u_{2},
\end{array} t>0, \mathbf{x} \in \Omega .\right.
$$

With the same boundary and initial conditions as (1.2) and (1.3), qualitative properties of nonnegative solutions of (1.4) have been intensively studied. The first remark is that the stable attractor of (1.4) consists of equilibrium solutions only (Hirsch [7] and Matano \& Mimura [12]). By this information, we may be concerned with existence and stability of non-negative equilibrium solutions for the study of asymptotic behavior of solutions.

Ecologically, let us assume the situation where two species are strongly competing, that is,

$$
a_{1} / b_{2}<r_{1} / r_{2}<b_{1} / a_{2}
$$

which indicates that stable constant equilibrium solutions are $\left(u_{1}, u_{2}\right)=\left(r_{1} / a_{1}, 0\right)$ and $\left(0, r_{2} / a_{2}\right)$ only. These solutions mean that only one of the competing species survives and the other is extinct. When $\Omega$ is convex, any non-constant equilibrium solutions are unstable, even if they exist (Kishimoto \& Weinberger [11]). In other words, stable equilibria are only $\left(r_{1} / a_{1}, 0\right)$ and $\left(0, r_{2} / a_{2}\right)$, which ecologically indicates that two strongly competing species can never coexist in any convex habitats. On the other hand, if the domain $\Omega$ is not convex, the structure of equilibrium solutions is not so simple but depends on the shape of $\Omega$. If $\Omega$ takes suitable dumb-bell shape, for instance, there exist stable non-constant equilibrium solutions, which exhibit spatial segregation of the two competing species in a sense that one region is nearly occupied by the species $U_{1}$, while the other is by $U_{2}$, that is, two competing species possibly coexist in suitably non-convex habitats [12]. Integrating the above, one finds that the asymptotic behavior of solutions of two competing species model (1.4) is rather clear, and that if the domain is convex, it is extremely simple.

On the other hand, for a multi-competing species case, the situation drastically changes. Even if we restrict ourselves to the diffusionless system of (1.1), the solution structures of the resulting O.D.E. system are complicated. There coexists a variety of periodically and aperiodically solutions, in addition to equilibria, depending on parameters $r_{i}$ and $a_{i j}$ (see [13], for example). There has been 
little work for the RD system (1.1) with $n \geqslant 3$, except for some results which are discussed by using singular perturbation methods $[9,10,14]$.

In this paper, we consider (1.1) with $n=3$, that is,

$$
\frac{\partial u_{i}}{\partial t}=D_{i} \Delta u_{i}+f_{i}\left(u_{1}, u_{2}, u_{3}\right) \quad(i=1,2,3), \quad t>0, \mathbf{x} \in \Omega,
$$

where $f_{i}=\left(r_{i}-a_{i 1} u_{1}-a_{i 2} u_{2}-a_{i 3} u_{3}\right) u_{i}(i=1,2,3)$, or simply

$$
\frac{\partial \mathbf{u}}{\partial t}=D \Delta \mathbf{u}+F(\mathbf{u}), \quad t>0, \mathbf{x} \in \Omega,
$$

where $\mathbf{u}=\left(u_{1}, u_{2}, u_{3}\right), D$ is the diagonal matrix with elements $\left\{D_{i}\right\}(i=1,2,3)$ and $F(\mathbf{u})=$ $\left(f_{1}(\mathbf{u}), f_{2}(\mathbf{u}), f_{3}(\mathbf{u})\right)$.

We first consider the diffusionless system of (1.6')

$$
\frac{\partial \mathbf{u}}{\partial t}=F(\mathbf{u}), \quad t>0
$$

assuming that $a_{i j}(i \neq j)$ are large comparing with other $a_{i i}$ to require that $P_{1}=\left(r_{1} / a_{11}, 0,0\right)$, $P_{2}=\left(0, r_{2} / a_{22}, 0\right)$ and $P_{3}=\left(0,0, r_{3} / a_{33}\right)$ are stable and other critical points are all unstable, that is, three species are in strong competition. It is shown in [20] that almost all non-negative solutions $\mathbf{u}(t)$ of (1.7) tend to one of $P_{i}(i=1,2,3)$. This ecologically implies that one of the competing species can survive and the other two are extinct. Under this non-coexistence situation, we assume that the diffusion rates $D_{i}$ are so sufficiently small, that is, $D_{i}=\epsilon^{2} d_{i}$ with a sufficiently small parameter $\epsilon$, where $d_{i}$ is some positive constant $(i=1,2,3)$. The resulting system from (1.6) is

$$
\frac{\partial \mathbf{u}}{\partial t}=\epsilon^{2} D^{\prime} \Delta \mathbf{u}+F(\mathbf{u}) \quad t>0, \mathbf{x} \in \Omega
$$

where $D^{\prime}$ is the diagonal matrix, with the initial and boundary conditions

$$
\mathbf{u}(0, \mathbf{x})=\mathbf{u}_{0}(\mathbf{x}) \geqslant 0, \mathbf{x} \in \Omega
$$

and

$$
\frac{\partial \mathbf{u}}{\partial v}=0 \quad t>0, \mathbf{x} \in \Omega .
$$

Since $\epsilon$ is sufficiently small, one can expect that the behavior of solutions of (1.8)-(1.10) essentially consists of two stages: The first stage is the occurrence of internal layer regions (when $\epsilon$ tends to zero, they become interfacial curves), which generally divide the domain $\Omega$ into three subdomains $\Omega_{1}, \Omega_{2}$ and $\Omega_{3}$ where the solution $\mathbf{u}(t, \mathbf{x})$ is close to one of $P_{1}, P_{2}$ and $P_{3}$. This indicates the appearance of spatial segregation of three competing species with triple junctions where three interfacial curves meet. The second stage is the motion of interfacial curves with triple junctions, that is, the dynamics of segregating patterns.

We demonstrate some numerical simulations of (1.8)-(1.10) in a rectangular domain $\Omega$. First, we show the occurrence of segregating patterns as the first stage in Figs 1a-1d. The domain $\Omega$ is clearly divided into three subdomains $\Omega_{1}, \Omega_{2}$ and $\Omega_{3}$, which are separated by interfacial curves with triple junctions. We consider the second stage. For the completely symmetric case where $d_{i}=d$, 
$a_{i i}=a, a_{i j}=b(i=1,2,3)$. The dynamics of segregating pattern changes slowly, as in Figs 1d$1 \mathrm{~g}$. We note that interfacial curves which are almost straight they move very slowly, and that angles between any two neighboring interfacial curves are equal. This angle condition can be intuitively understood by the fact that three competing species possess completely symmetric property. We next consider non-symmetric cases. The first example is a semi-symmetric case where $d_{i}=d$ $(i=1,2,3), a_{12}=a_{21}=b_{1}, a_{23}=a_{32}=b_{2}$ and $a_{31}=a_{13}=b_{3}$ but $b_{1}, b_{2}$ and $b_{3}$ are not necessarily equal, that is, $120^{\circ}$. As in Fig. 2, the dynamics of a segregating pattern is qualitatively similar to the above symmetric case except for the angles at triple junction areas, which seem to be not necessarily equal. The second is the case with ordering property where $a_{12}<a_{21}, a_{13}<a_{31}$ and $a_{23}<a_{32}$, that is, the species $U_{1}$ is the strongest of the three. As in Fig. 3, the dynamics of pattern is much faster than the previous two cases and, as is easily expected, the domain is gradually occupied by the strongest species $U_{1}$. The third is a symmetrically cyclic case where $a_{12}=a_{23}=a_{31}=b$ and $a_{21}=a_{32}=a_{13}=b^{\prime}$ with $b \neq b^{\prime}$. As the cyclic property suggests, there appear stationary rotating spiral patterns with three arms, as in Fig. 4. Finally, the fourth is a general case with cyclic property where $a_{12}<a_{21}, a_{23}<a_{32}$ and $a_{31}<a_{13}$. As in Fig. 5, there is no longer any stationary rotating spirals but complex spatio-temporal patterns with several clustering spirals, where each spiral seems to be steadily rotating in a vicinity of triple junction areas.

We have observed qualitatively different behaviors of interfaces with triple junctions, depending on values of interspecific competition rates. These results suggest several aspects on segregating patterns of three competing species, which are drastically different from ones of two competing species: (i) even if $\Omega$ is convex domain in $\mathbf{R}^{2}$, it is possible for the three species to coexist; (ii) although the behavior of solutions to the diffusionless system (1.7) is so simple, solutions of the corresponding RD system (1.8) exhibit complicated spatio-temporal coexisting patterns; (iii) there appear rich phenomena on motion of interfaces with triple junctions, comparing with the ones in other gradient systems including vector-valued Ginzburg-Landau equations with three well potentials [17].

The paper is organized as follows. In Section 2, we give some preliminaries for a two competing species model. In Section 3, we numerically study the dynamics of interfacial curves of (1.8), depending on values of $d_{i}$ and $a_{i j}(i, j=1,2,3)$. In Section 4 , we derive an angle condition at triple junction points by a formal asymptotic expansion. Finally in Section 5, we give some remarks on the results obtained in Sections 3 and 4.

\section{Preliminaries}

In this section, we briefly mention the known result on the dynamics of interfaces arising in the two competing species system (1.4) as preliminaries of our study.

We consider (1.4) on the whole plane $\mathbf{R}^{2}$ and write it again as

$$
\left\{\begin{array}{l}
\frac{\partial v}{\partial t}=\epsilon^{2} d_{v} \Delta v+\left(r_{c}-a_{v} v-b_{v} w\right) v, \\
\frac{\partial w}{\partial t}=\epsilon^{2} d_{w} \Delta w+\left(r_{w}-b_{w} v-a_{w} w\right) w,
\end{array} \quad t>0, \mathbf{x} \in \mathbf{R}^{2},\right.
$$

where all of the coefficients are positive constants. We assume

$$
a_{v} / b_{w}<r_{v} / r_{w}<b_{v} / a_{w} .
$$


Then, as was noted in the previous section, $\tilde{P}_{v}=\left(r_{v} / a_{v}, 0\right)$ and $\tilde{P}_{w}=\left(0, r_{w} / a_{w}\right)$ are two stable constant equilibria of (2.1).

Since $\epsilon$ is sufficiently small, there occurs a sharp interface $\Gamma$ which divides $\mathbf{R}^{2}$ into two subdomains $\Omega_{v}$ and $\Omega_{w}$ on which the solution $(v, w)$ is close to $\tilde{P}_{v}$ and $\tilde{P}_{w}$, respectively.

Let $\Phi(s)=(\phi(s), \psi(s))(s=x-\theta t)$ be a one-dimensional traveling front solution of (2.1) with velocity $\theta$, connecting two stable equilibrium states $\tilde{P}_{v}$ and $\tilde{P}_{w}$. Then it satisfies

$$
\begin{aligned}
& \left\{\begin{array}{l}
0=d_{v} \phi_{s s}+\theta \phi_{s}+\left(r_{v}-a_{v} \phi-b_{v} \psi\right) \phi \\
0=d_{w} \psi_{s s}+\theta \psi_{s}+\left(r_{w}-a_{w} \psi-b_{w} \phi\right) \psi
\end{array}-\infty<s<\infty,\right. \\
& \Phi(-\infty)=\tilde{P}_{v}, \Phi(+\infty)=\tilde{P}_{w} .
\end{aligned}
$$

The existence and uniqueness of the solution $(\Phi(s), \theta)$ were proved by Kan-on [8]. The equation of the interface $\Gamma(t)$ is given by Ei \& Yanagida [4]. Let $V$ be the normal velocity oriented from $\Omega_{v}$ to $\Omega_{w}$. When $\theta \neq 0$

$$
V=\epsilon \theta,
$$

while, when $\theta=0$,

$$
V=-\epsilon^{2} L \kappa
$$

where $\kappa$ is the mean curvature of the interface $\Gamma$. The constant $L$ is given as follows: let $\Phi^{0}(s)=$ $\left(\phi^{0}(s), \psi^{0}(s)\right)$ be a solution of $(2.3)$ with $\theta=0$. Then $\Phi_{s}^{0}=\left(\phi_{s}^{0}(s), \psi_{s}^{0}(s)\right)$ is the eigenfunction corresponding to zero eigenvalue of the linearized operator around $\Phi^{0}$. Let $\left(\phi^{*}(s), \psi^{*}(s)\right)$ be the eigenfunction corresponding to zero eigenvalue of its adjoint operator. Now $L$ is represented as

$$
L=\frac{\int_{-\infty}^{\infty}\left(d_{v} \phi_{s}^{0}(s) \phi^{*}(s)+d_{w} \psi_{s}^{0}(s) \psi^{*}(s)\right) \mathrm{d} s}{\int_{-\infty}^{\infty}\left(\phi_{s}^{0}(s) \phi^{*}(s)+\psi_{s}^{0}(s) \psi^{*}(s)\right) \mathrm{d} s}>0
$$

Thus, the interfacial equation derived from (2.1) with a sufficiently small $\epsilon$ can be generally described by

$$
V=\epsilon \theta-\epsilon^{2} L \kappa
$$

and the profile of $(v(t, \mathbf{x}), w(t, \mathbf{x}))$ in the neighborhood of $\Gamma$ is close to $\Phi(\operatorname{dist}(\mathbf{x}, \Gamma) / \epsilon)$.

\section{Dynamics of interfacial curves}

As in Fig. 6, let $\Gamma_{1}$ be an interfacial curve between $\Omega_{2}$ and $\Omega_{3}$, and $\Gamma_{2}$ and $\Gamma_{3}$ are similarly defined, and let $\gamma$ be the position of a triple junction at which three curves $\Gamma_{i}(i=1,2,3)$ meet.

First, we consider the behavior of solutions $\mathbf{u}(t, \mathbf{x})$ which are apart from the triple junction $\gamma$. In order to do it, we may consider it only in a neighborhood of one of the interfaces, say $\Gamma_{1}$, where $\mathbf{u}(t, \mathbf{x})$ changes rapidly from $P_{2}$ to $P_{3}$ and the $u_{1}$-component of $\mathbf{u}$ is almost identically zero. Therefore, one can expect that the dynamics of $\Gamma_{1}$ is approximately described by 


$$
V_{1}=\epsilon \theta_{1}-\epsilon^{2} L_{1} \kappa_{1},
$$

where $V_{1}$ and $\kappa_{1}$ denote the normal velocity measured from $\Omega_{2}$ to $\Omega_{3}$ and the curvature of the interface $\Gamma_{1}$ respectively, and $\theta_{1}$ is the velocity of the one-dimensional traveling front solution $\left(\phi^{1}(s), \psi^{1}(s)\right)$ which satisfies

$$
\begin{aligned}
& \left\{\begin{array}{l}
0=d_{2} \phi_{s s}^{1}+\theta_{1} \phi_{s}^{1}+\left(r_{2}-a_{22} \phi^{1}-a_{23} \psi^{1}\right) \phi^{1} \\
0=d_{3} \psi_{s s}^{1}+\theta_{1} \psi_{s}^{1}+\left(r_{3}-a_{33} \psi^{1}-a_{32} \phi^{1}\right) \psi^{1}-\infty<s<\infty,
\end{array}\right. \\
& \left(\phi^{1}, \psi^{1}\right)(-\infty)=\tilde{P}_{2},\left(\phi^{1}, \psi^{1}\right)(+\infty)=\tilde{P}_{3} .
\end{aligned}
$$

Then, it turns out that the profile $\left(u_{2}, u_{3}\right)$ in the neighborhood of $\Gamma_{1}$ is close to $\left(\phi^{1}, \psi^{1}\right)\left(\operatorname{dist}\left(\mathbf{x}, \Gamma_{1}\right) / \epsilon\right)$.

Putting $\Phi^{1}(s)=\left(0, \phi^{1}(s), \psi^{1}(s)\right)$, we see that $\Phi^{1}(s)$ satisfies

$$
0=D \Phi_{s s}^{1}+\theta_{1} \Phi_{s}^{1}+F\left(\Phi^{1}\right) \quad(-\infty<s<\infty)
$$

with

$$
\Phi^{1}(-\infty)=\left(0, r_{2} / a_{22}, 0\right)=P_{2}, \quad \Phi^{1}(+\infty)=\left(0,0, r_{3} / a_{33}\right)=P_{3},
$$

and that the profile of $\mathbf{u}$ in the neighborhood of $\Gamma_{1}$ is close to $\Phi^{1}\left(\operatorname{dist}\left(\mathbf{x}, \Gamma_{1}\right) / \epsilon\right)$.

As was already mentioned in Section 2, the constant $L_{1}$ in (3.1) is represented as

$$
L_{1}=\frac{\int_{-\infty}^{+\infty}\left(d_{2} \phi_{s}(s) \phi^{*}(s)+d_{3} \psi_{s}(s) \psi^{*}(s)\right) \mathrm{d} s}{\int_{-\infty}^{\infty}\left(\phi_{s}(s) \phi^{*}(s)+\psi_{s}(s) \psi^{*}(s)\right) \mathrm{d} s},
$$

where $(\phi(s), \psi(s))$ is a solution of (3.2) with $\theta_{1}=0$. Let $K$ be the linearized operator around $(\phi(s), \psi(s))$ and $K^{*}$ be its adjoint operator. $\left(\phi_{s}(s), \psi_{s}(s)\right)$ is the eigenfunction corresponding to the zero eigenvalue of $K$ and $\Phi^{*}(s)=\left(\phi^{*}(s), \psi^{*}(s)\right)$ is the eigenfunction corresponding to zero eigenvalue of $K^{*}$. Let $\Psi^{1}(s)=(0, \phi(s), \psi(s))$ and $\Psi^{1, *}(s)=\left(0, \phi^{*}(s), \psi^{*}(s)\right)$. Then $L_{1}$ is represented as

$$
L_{1}=\frac{\int_{-\infty}^{\infty}\left\langle D \Psi_{s}^{1}(s), \Psi^{1, *}(s)\right\rangle \mathrm{d} s}{\int_{-\infty}^{\infty}\left\langle\Psi_{s}^{1}(s), \Psi^{1, *}(s)\right\rangle \mathrm{d} s},
$$

where $\langle$,$\rangle is an inner product of vectors in \mathbf{R}^{3}$.

By using the similar discussion to (3.1), the dynamics of $\Gamma_{2}$ and $\Gamma_{3}$ are approximately given by

$$
V_{i}=\epsilon \theta_{i}-\epsilon^{2} L_{i} \kappa_{i} \quad(i=2,3),
$$

where $V_{i}, \theta_{i}$ and $\kappa_{i}$ are similarly defined and $L_{i}$ is given by

$$
L_{i}=\frac{\int_{-\infty}^{\infty}\left\langle D \Psi_{s}^{i}(s), \Psi^{i, *}(s)\right\rangle \mathrm{d} s}{\int_{-\infty}^{\infty}\left\langle\Psi_{s}^{i}(s), \Psi^{i, *}(s)\right\rangle \mathrm{d} s},
$$


(a) 2

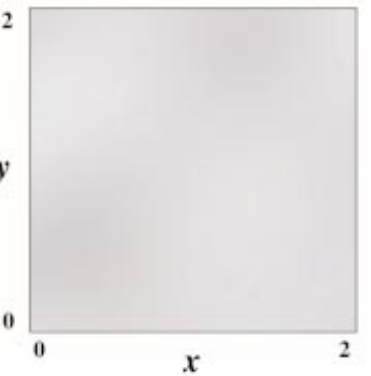

(b)

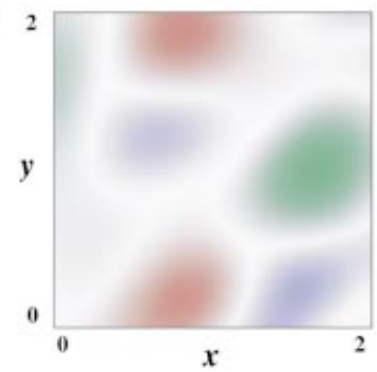

(d) 2

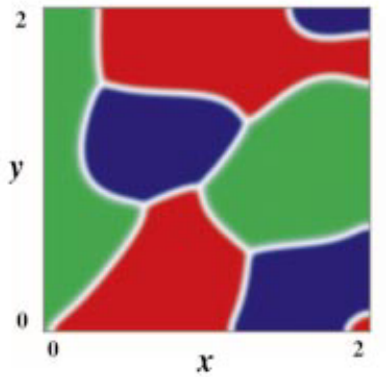

(f)

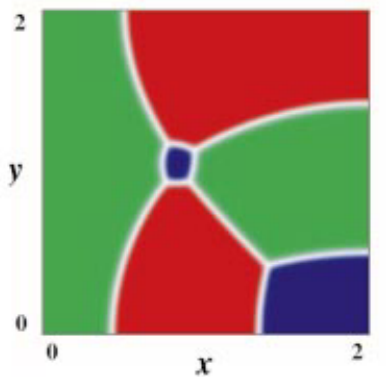

$u_{1}$

$u_{2}$

$u_{3}$

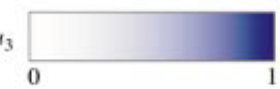

(c)

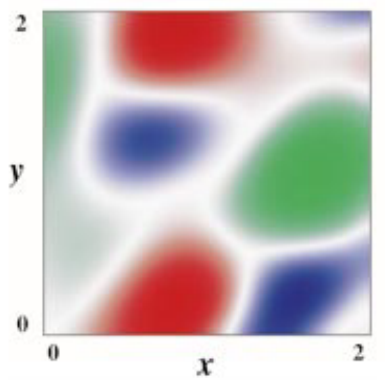

(e)

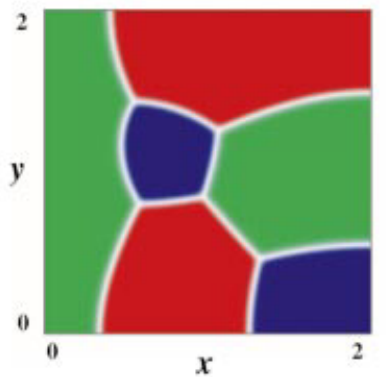

(g)

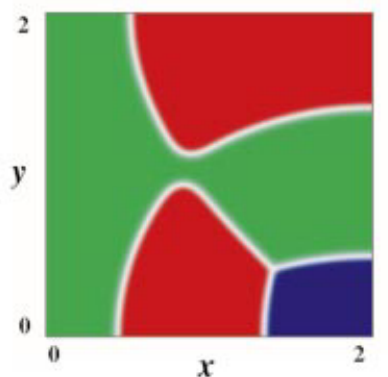

FIG. 1. Appearance of phase separation and dynamics of interfaces with triple junctions in (1.8)-(1.10) where $\epsilon=1.0 \times 10^{-2}, d_{i}=1.0, r_{i}=1.0, a_{i i}=1.0, a_{i j}=3.0(i \neq j)(i, j=1,2,3)$. The initial conditions are $u_{0 i}(x)=0.143$ with small fluctuations. (a) $t=0$, (b) $t=2.5$, (c) $t=5$, (d) $t=25$, (e) $t=1225$, (f) $t=2425,(\mathrm{~g}) t=2625$. 

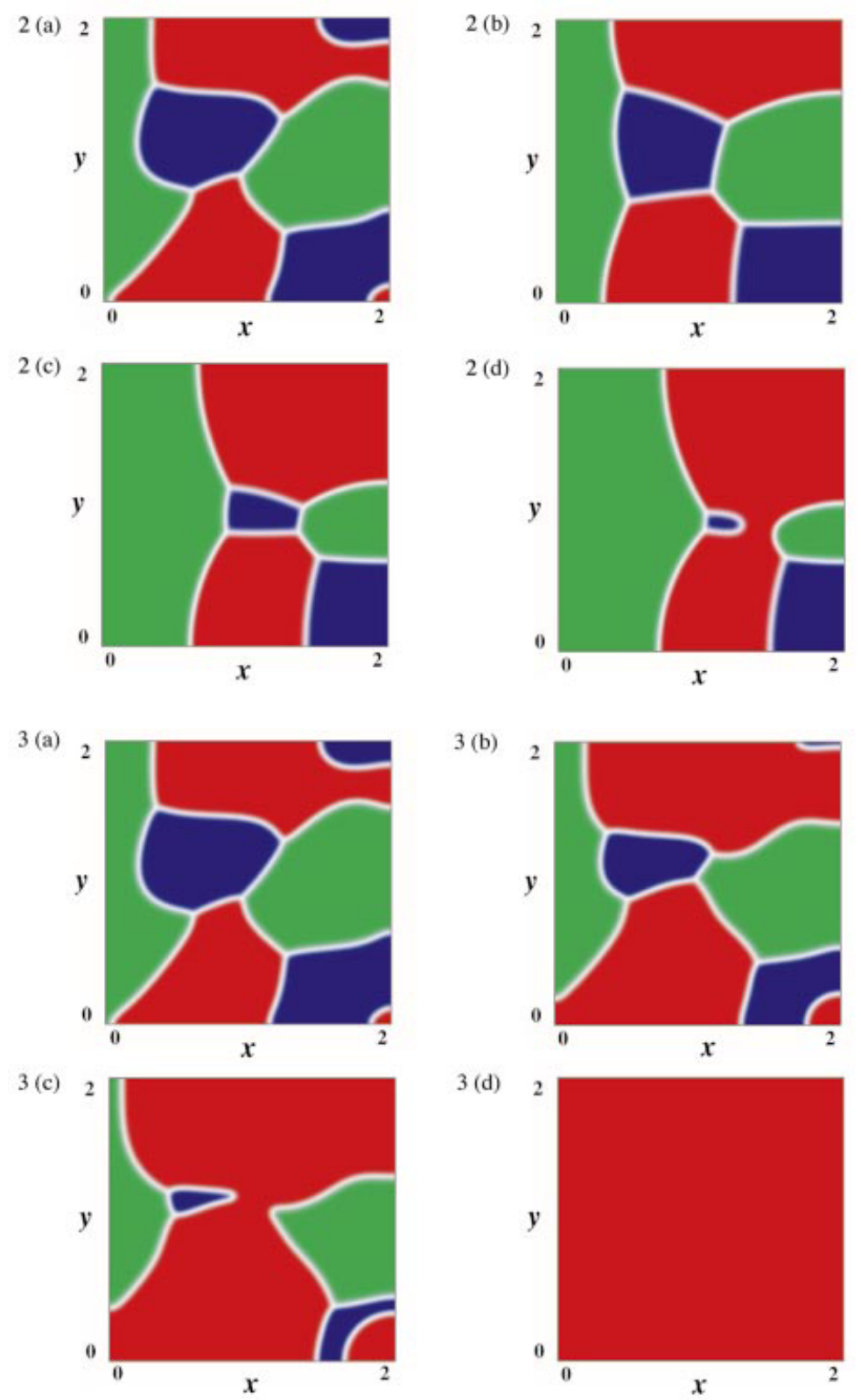

FIG. 2. Dynamics of interfaces with triple junctions in (1.8)-(1.10) where the parameters are the same as those in Fig. 1 except for $a_{12}=a_{21}=8.0, a_{23}=a_{32}=4.0$ and $a_{31}=a_{13}=2.0$. (a) $t=0$, (b) $t=1200$, (c) $t=6000$, (d) $t=7250$.

FIG. 3. Dynamics of interfaces under situation where $U_{1}$ is stronger than $U_{2}$ and $U_{3}$. The parameters are the same as those in Fig. 1 except for $a_{12}=2.0, a_{13}=3.0, a_{21}=3.0, a_{23}=3.0, a_{31}=5.0, a_{32}=4.0$. (a) $t=0$, (b) $t=50$, (c) $t=100$, (d) $t=300$. 

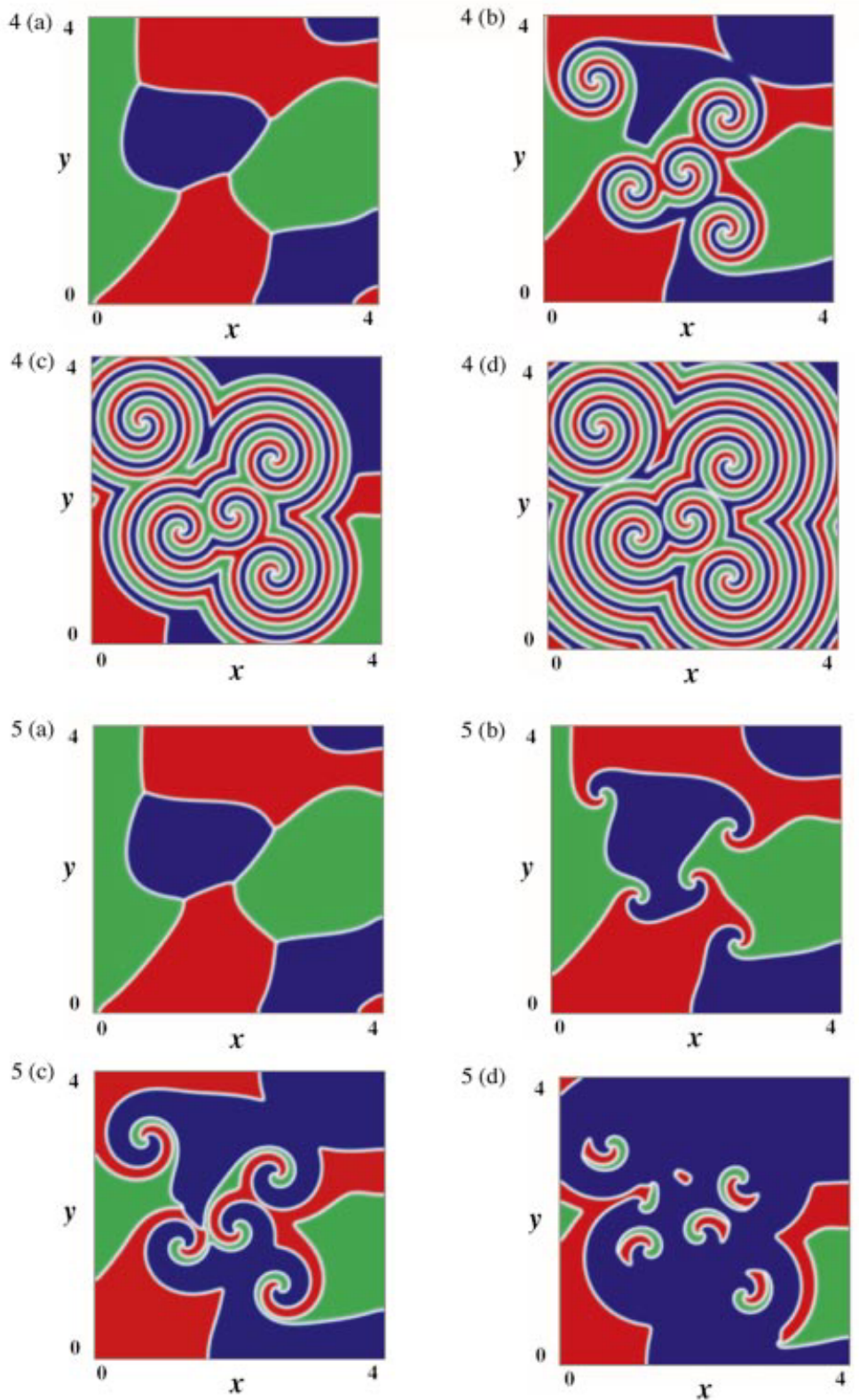

FIG. 4. Dynamics of stationary rotating spirals with three arms in (1.8)-(1.10) where the parameters are the same as those in Fig. 1 except for $a_{12}=a_{23}=a_{31}=2.0, a_{21}=a_{13}=a_{32}=7.0$. (a) $t=0$, (b) $t=100$, (c) $t=200,(\mathrm{~d}) t=300$.

FIG. 5. Dynamics of clustering spirals in (1.8)-(1.10) where the parameters are the same as those in Fig. 1 except for $a_{12}=3.0, a_{21}=6.5, a_{13}=6.0, a_{31}=2.9, a_{23}=3.5, a_{32}=6.1$. (a) $t=0$, (b) $t=100$, (c) $t=200,(\mathrm{~d}) t=300$ 


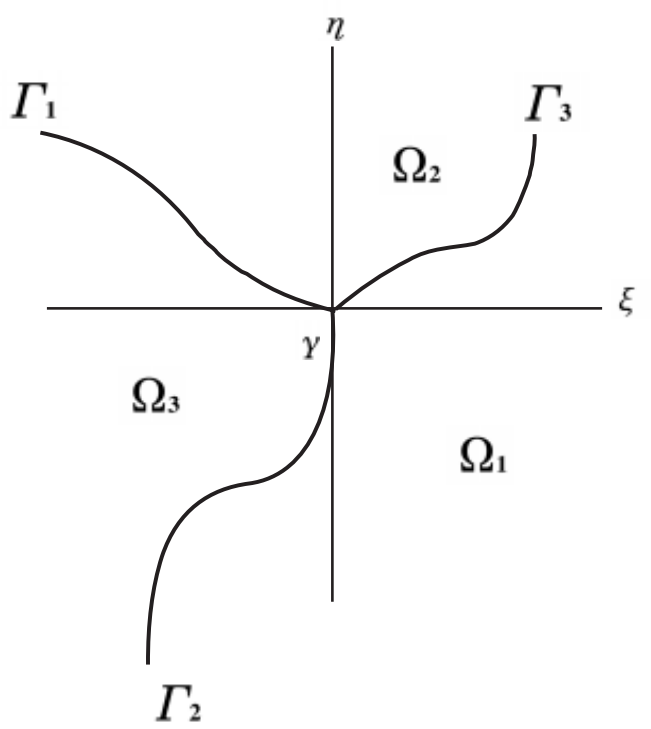

FIG. 6. Three phases $\Omega_{1}, \Omega_{2}$ and $\Omega_{3}$ with a triple juction $\gamma$.

where $\Psi^{i}(s)$ and $\Psi^{i, *}(s)(i=2,3)$ are defined similarly to $\Psi^{1}(s)$ and $\Psi^{1, *}(s)$.

We may say that if $\theta_{1}=0$, two species $U_{2}$ and $U_{3}$ are equal in competition, if $\theta_{1}>0, U_{2}$ is competitively stronger than $U_{3}$ and if $\theta_{1}<0$, vice versa.

The relations among $\theta_{1}, \theta_{2}$ and $\theta_{3}$ are essentially classified into four cases:

Case I: $\theta_{1}=\theta_{2}=\theta_{3}=0$.

Case II: $\theta_{1}=0, \theta_{2}>0$ and $\theta_{3}<0$.

Case III: $\theta_{2}<0$ and $\theta_{3}>0\left(U_{1}\right.$ is the strongest of the three).

Case IV: $\theta_{i}>0(i=1,2,3)\left(U_{1}\right.$ is stronger than $U_{2}, U_{2}$ is stronger than $U_{3}$ and $U_{3}$ is stronger than $U_{1}$, in other words, there is a cyclic property among them).

Other cases are reduced to one of the four cases by appropriately exchanging species with each other.

For Case II, the whole domain $\Omega$ is first occupied by $\Omega_{2}$ and $\Omega_{3}$ and eventually by either $\Omega_{2}$ or $\Omega_{3}$. For Case III, as was shown in Fig. 3, the domain $\Omega_{1}$ expands, and eventually it occupies the whole domain $\Omega$. For Case IV, because of cyclic property, one can expect that certain rotating behavior appears in the dynamics of interfaces. The resulting patterns crucially depend on the relation among $\theta_{1}, \theta_{2}$ and $\theta_{3}$, as was shown in Figs 4 and 5. For Case I (or even if $\theta_{i}(i=1,2,3)$ are not zero but sufficiently small), one can expect that the interfaces move very slowly by essentially curvature effect only. However, if triple junctions appear, the information on two competing case give nothing on dynamics of interfaces.

In the next section, we will study the dynamics of interfaces with triple junctions under this situation. 


\section{Angle condition at the triple junction point $\gamma$}

We consider the situation that all $\theta_{i}$ are of order $O(\epsilon)$, that is, $\theta_{i}=\epsilon c_{i}$ for constant $c_{i}(i=1,2,3)$. Then, the interfacial equation (3.6) becomes

$$
V_{i}=\epsilon^{2}\left(c_{i}-L_{i} \kappa_{i}\right)(i=1,2,3) .
$$

In order to realize this situation, we assume that $F(\mathbf{u})$ depends on $\epsilon$, and express $F(\mathbf{u})=F(\mathbf{u} ; \epsilon)$, satisfying $F_{0}(\mathbf{u})=F(\mathbf{u} ; 0)$. The resulting system is

$$
\frac{\partial \mathbf{u}}{\partial t}=D \Delta \mathbf{u}+F(\mathbf{u} ; \epsilon)
$$

Let $P_{i}(\epsilon)$ be the constant equilibria corresponding to $P_{i}$ given in Section 1 , satisfying $P_{i}^{0}=$ $P_{i}(0) \quad(i=1,2,3)$. Then, our assumption on $F_{0}(\mathbf{u})$ is that there is a solution $\Psi^{1}(s)$ satisfying the equation

$$
0=D \Psi_{s s}+F_{0}(\Psi) \quad-\infty<s<\infty
$$

with $\Psi(-\infty)=P_{2}^{0}$ and $\Psi(\infty)=P_{3}^{0}$. Let $\Phi^{1}(s ; \epsilon)$ be a one-dimensional traveling front solutions of (3.3) with velocity $\epsilon \theta_{1}$, connecting $P_{2}(\epsilon)$ and $P_{3}(\epsilon)$, satisfying $\Psi^{1}(s)=\lim _{\epsilon \downarrow 0} \Phi^{1}(s ; \epsilon)$. We also give the similar assumption on $\Phi^{i}(s ; \epsilon)(i=2,3)$. Under this situation, we shall show that three interfaces $\Gamma_{i}(i=1,2,3)$ which meet at a triple junction point $\gamma$ satisfy a certain angle condition.

With $T=\epsilon^{2} t$, we notice by (4.1) that the movement of interfaces $\Gamma_{i}$ are in time-scale $T$. Thus, we may suppose:

(H1) the triple junction point $\gamma$ moves in time-scale $T$, that is, $\gamma=\gamma(T)$.

On the other hand, we already know that the profile of the solution $\mathbf{u}$ in the neighborhood of $\Gamma_{1}$ is close to $\Phi^{1}\left(\operatorname{dist}\left(\mathbf{x}, \Gamma_{1}\right) / \epsilon\right)$, as was stated in Section 3. Since (4.1) and (H1) indicate that the time change of $\operatorname{dist}\left(\mathbf{x}, \Gamma_{1}\right)$ is in time-scale $T$, we know

$$
\begin{aligned}
\mathbf{u}_{t} & \sim \Phi_{s}^{1}\left(\operatorname{dist}\left(\mathbf{x}, \Gamma_{1}\right) / \epsilon\right) \times \frac{\partial \operatorname{dist}\left(\mathbf{r}, \Gamma_{1}\right)}{\partial t} / \epsilon \\
& =O\left(\epsilon^{2}\right) / \epsilon \\
& =O(\epsilon),
\end{aligned}
$$

and then may suppose:

(H2) $\mathbf{u}$ changes in time-scale $\tau=\epsilon t$, that is, $\mathbf{u}=\mathbf{u}(\tau, \mathbf{x})$.

By transforming the coordinates $\mathbf{x}=(x, y)$ to $(\xi, \eta)$ with $(x, y)=\gamma+\epsilon(\xi, \eta)$ and using $\mathbf{v}(t, \xi, \eta)=\mathbf{u}(t, \gamma(t)+\epsilon(\xi, \eta))$, in view of (H1), Eqn (4.3) is rewritten as

$$
\begin{aligned}
\mathbf{v}_{t} & =D \Delta \mathbf{v}+F(\mathbf{v} ; \epsilon)+\frac{1}{\epsilon} \nabla \mathbf{v} \cdot \gamma_{t} \\
& =D \Delta \mathbf{v}+F(\mathbf{v} ; \epsilon)+\epsilon \nabla \mathbf{v} \cdot \gamma_{T},
\end{aligned}
$$

where $\cdot$ denotes an operation of $\nabla \mathbf{v}$ on $\gamma_{T}$. We note by (H2) that $\mathbf{v}$ can be written as $\mathbf{v}=\mathbf{v}(\tau, \xi, \eta)$. 


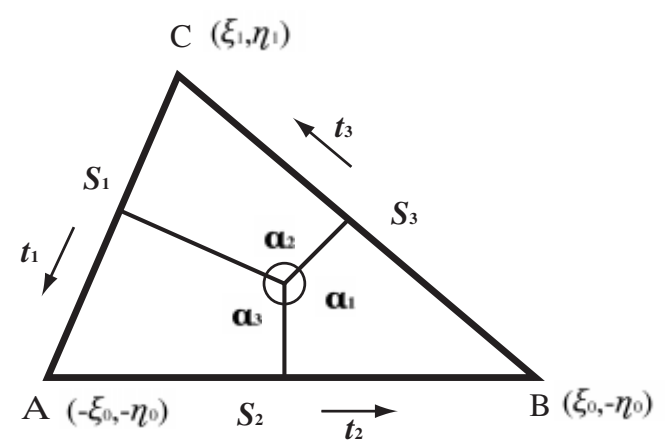

FIG. 7. A triple juction with angles $\alpha_{1}, \alpha_{2}$ and $\alpha_{3}$.

Expanding $\mathbf{v}$ as

$$
\mathbf{v}=\mathbf{v}^{0}+\epsilon \mathbf{v}^{1}+\cdots
$$

and substituting it into (4.4), we have

$$
0=D \Delta \mathbf{v}^{0}+F_{0}\left(\mathbf{v}^{0}\right),
$$

which is the lowest order equation of (4.5) with respect to $\epsilon$.

Let $v_{1}$ be the unit vector in the direction toward the tangential direction of the interface $\Gamma_{1}$ at the triple junction point $\gamma$ and let $\alpha_{1}$ be an angle between $\nu_{2}$ and $\nu_{3} . \alpha_{2}$ and $\alpha_{3}$ are similarly defined. First, we assume that all angles $\alpha_{i}(i=1,2,3)$ are larger than $\pi / 2$.

For convenience, we rotate the new coordinates $(\xi, \eta)$ in such a way that the direction $v_{2}$ coincides with the $\eta$-axis to the negative direction, as in Fig. 6 . Then, $v_{i}(i=1,2,3)$ are given by

$$
\left\{\begin{array}{l}
v_{1}=\left(-\sin \alpha_{3},-\cos \alpha_{3}\right), \\
v_{2}=(0,-1), \\
v_{3}=\left(\sin \alpha_{1},-\cos \alpha_{1}\right) .
\end{array}\right.
$$

We now take a sufficiently large triangle with three apexes $A=\left(-\xi_{0},-\eta_{0}\right), B=\left(\xi_{0},-\eta_{0}\right)$ with sufficiently large positive constants $\xi_{0}$ and $\eta_{0}$, and $C=\left(\xi_{1}, \eta_{1}\right)$, which is an intersection of half lines starting at $A$ and $B$ perpendicular to $v_{1}$ and $\nu_{3}$. Let $S_{i}, \mathbf{t}_{i}(i=1,2,3)$ be the sides $C A, A B$ and $B C$, and their tangential unit vectors in the directions of $\overrightarrow{C A}, \overrightarrow{A B}$ and $\overrightarrow{B C}$, respectively, as in Fig. 7. Since $v_{i}$ is the outward normal unit vectors of the triangle $\triangle A B C$ on sides of $S_{i}(i=1,2,3)$, and $\mathbf{t}_{i}(i=1,2,3)$ are explicitly given by

$$
\left\{\begin{array}{l}
\mathbf{t}_{1}=\left(\cos \alpha_{3},-\sin \alpha_{3}\right), \\
\mathbf{t}_{2}=(1,0), \\
\mathbf{t}_{3}=\left(\cos \alpha_{1}, \sin \alpha_{1}\right) .
\end{array}\right.
$$

We now introduce the coordinates $\left(\xi_{i}, \eta_{i}\right)$ with respect to vectors $v_{i}$ and $\mathbf{t}_{i}$ by

$$
(\xi, \eta)=\xi_{i} \mathbf{t}_{i}+\eta_{i} v_{i} \quad(i=1,2,3) .
$$


Then, the sides $S_{i}$ are represented as

$$
S_{i}=\left\{\xi_{i} \mathbf{t}_{i}+\eta_{i}^{0} v_{i} ; \xi_{i}^{-} \leqslant \xi \leqslant \xi_{i}^{+}\right\}
$$

for positive constants $\xi_{i}^{-}, \xi_{i}^{+}$and $\eta_{i}^{0}(i=1,2,3)$, which satisfy

$$
\begin{aligned}
\xi_{2}^{ \pm} & = \pm \xi_{0}, \\
\eta_{2}^{0} & =\eta_{0}, \\
A & =\xi_{1}^{+} \mathbf{t}_{1}+\eta_{1}^{0} v_{1}=\xi_{2}^{-} \mathbf{t}_{2}+\eta_{2}^{0} v_{2}, \\
B & =\xi_{2}^{+} \mathbf{t}_{2}+\eta_{2}^{0} \nu_{2}=\xi_{3}^{-} \mathbf{t}_{3}+\eta_{3}^{0} \nu_{3}, \\
C & =\xi_{3}^{+} \mathbf{t}_{3}+\eta_{3}^{0} \nu_{3}=\xi_{1}^{-} \mathbf{t}_{1}+\eta_{1}^{0} v_{1} .
\end{aligned}
$$

We take the triangle $\triangle A B C$ large enough such that all $\eta_{i}^{0}$ and $\xi_{i}^{ \pm}$are sufficiently large and define the inside region of $\triangle A B C$ by $R$.

Now, we note that $\mathbf{v}^{0}$ satisfies

$$
\mathbf{v}^{0} \rightarrow \Psi^{i} \text { as } \eta_{i} \rightarrow \infty
$$

that is,

$$
\mathbf{v}^{0}(\xi, \eta) \rightarrow \Psi^{i}\left(\xi_{i}\right) \text { as } \eta_{i} \rightarrow \infty
$$

Hence, we may suppose

$$
\left\{\begin{aligned}
\mathbf{v}^{0}(\xi, \eta) & \simeq \Psi^{i}\left(\xi_{i}\right), \\
\frac{\partial \mathbf{v}^{0}}{\partial \eta_{i}} & \simeq 0
\end{aligned}\right.
$$

on the sides $S_{i}$, since $\eta_{i}^{0}$ are sufficiently large. Here, we use the notation $a \simeq b$ in the sense that $|a-b| \rightarrow 0$ when all $\eta_{i}^{0}$ and $\xi_{i}^{ \pm}$go to infinity.

Let $\Xi_{1}(\eta)$ and $\Xi_{3}(\eta)\left(-\eta_{0} \leqslant \eta \leqslant \eta_{1}\right)$ be values of the $\xi$-coordinate of the point $(\xi, \eta) \in$ $S_{i}(i=1,3)$, that is,

$$
\Xi_{1}(\eta)=\frac{\eta+\eta_{1}^{0}}{\tan \left(\pi-\alpha_{3}\right)}-\xi_{0} \quad \text { and } \quad \Xi_{3}(\eta)=\xi_{0}-\frac{\eta+\eta_{3}^{0}}{\tan \left(\pi-\alpha_{1}\right)}
$$

Since $\Psi^{i}$ satisfies (4.3), we have

$$
0=\frac{1}{2}\left\langle D \Psi_{s}^{i}, \Psi_{s}^{i}\right\rangle_{s}+\left\langle F_{0}\left(\Psi^{i}\right), \Psi_{s}^{i}\right\rangle
$$

and integrating this from $-\infty$ to $s$,

$$
\int_{-\infty}^{s}\left\langle F_{0}\left(\Psi^{i}\right), \Psi_{s}^{i}\right\rangle \mathrm{d} s=-\frac{1}{2}\left\langle D \Psi_{s}^{i}, \Psi_{s}^{i}\right\rangle
$$


and then

$$
\begin{aligned}
\int_{-\infty}^{\infty} \int_{-\infty}^{s}\left\langle F_{0}\left(\Psi^{i}\left(s^{\prime}\right)\right), \Psi_{s}^{i}\left(s^{\prime}\right)\right\rangle \mathrm{d} s^{\prime} \mathrm{d} s & =\int_{-\infty}^{\infty}\left\langle D \Psi_{s}^{i}(s), \Psi_{s}^{i}(s)\right\rangle \mathrm{d} s \\
& =-\frac{1}{2} M_{i} .
\end{aligned}
$$

We consider the quantity

$$
\iint_{R}\left(\int_{\Xi_{1}(\eta)}^{\xi}\left\langle F_{0}\left(\mathbf{v}^{0}\right), \mathbf{v}_{\xi}^{0}\right\rangle \mathrm{d} \xi^{\prime}\right)_{\eta} \mathrm{d} \xi \mathrm{d} \eta
$$

Let $v=\left(\nu^{\xi}, v^{\eta}\right)$ be the outward normal unit vector of $\triangle A B C$ along its sides $\partial R=S_{1} \cup S_{2} \cup S_{3}$. Since $v=v_{i}$ on the side $S_{i}$, Green's formula rewrites (4.14) as

$$
\int_{\partial R} \int_{\Xi_{1}(\eta)}^{\xi}\left\langle F_{0}\left(\mathbf{v}^{0}\right), \mathbf{v}_{\xi}^{0}\right\rangle \mathrm{d} \xi^{\prime} v^{\eta} \mathrm{d} s
$$

where $s$ denotes an arclength parameter on the boundary $\partial R$. We divide this into the following three parts:

$$
\int_{S_{1} \cup S_{2} \cup S_{3}} \int_{\Xi_{1}(\eta)}^{\xi}\left\langle F_{0}\left(\mathbf{v}^{0}\right), \mathbf{v}_{\xi}^{0}\right\rangle \mathrm{d} \xi^{\prime} v^{\eta} \mathrm{d} s=K_{1}+K_{2}+K_{3}
$$

On the side $S_{1}$, we know

$$
\int_{\Xi_{1}(\eta)}^{\xi}\left\langle F_{0}\left(\mathbf{v}^{0}\right), \mathbf{v}_{\xi}^{0}\right\rangle \mathrm{d} \xi^{\prime}=0
$$

because $(\xi, \eta) \in S_{1}$ means $\xi=\Xi_{1}(\eta)$. Hence, we have

$$
K_{1}=0 .
$$

On the side $S_{2}$, we know $v=v_{2}=(0,-1), s=\xi_{2}$ and $\mathbf{v}^{0} \simeq \Psi^{2}\left(\xi_{2}\right)$ by (4.6) and (4.11). Hence, by (4.12) and (4.13), we have

$$
\begin{aligned}
K_{2} & \simeq \int_{S_{2}} \int_{-\xi_{0}}^{s}\left\langle F_{0}\left(\Psi^{2}\left(\xi^{\prime}\right), \Psi_{s}^{2}\left(\xi^{\prime}\right)\right\rangle \mathrm{d} \xi^{\prime}(-1) \mathrm{d} s\right. \\
& \simeq-\int_{-\infty}^{\infty} \int_{-\infty}^{s}\left\langle F_{0}\left(\Psi^{2}\left(\xi^{\prime}\right), \Psi_{s}^{2}\left(\xi^{\prime}\right)\right\rangle \mathrm{d} \xi^{\prime} \mathrm{d} s\right. \\
& =\frac{1}{2} M_{2} .
\end{aligned}
$$

On the side $S_{3}$, by $v=\left(\sin \alpha_{1},-\cos \alpha_{1}\right)$, d $s=\frac{1}{\sin \alpha_{1}} \mathrm{~d} \eta$ and $\xi=\Xi_{3}(\eta) K_{3}$ is represented as

$$
K_{3}=\int_{S_{3}} \int_{\Xi_{1}(\eta)}^{\Xi_{3}(\eta)}\left\langle F_{0}\left(\mathbf{v}^{0}\right), \mathbf{v}_{\xi}^{0}\right\rangle \mathrm{d} \xi^{\prime}\left(-\cos \alpha_{1}\right) \mathrm{d} s
$$




$$
\begin{aligned}
& =-\frac{1}{\tan \alpha_{1}} \int_{-\eta_{0}}^{\eta_{1}} \int_{\Xi_{1}(\eta)}^{\Xi_{3}(\eta)}\left\langle F_{0}\left(\mathbf{v}^{0}\right), \mathbf{v}_{\xi}^{0}\right\rangle \mathrm{d} \xi \mathrm{d} \eta \\
& =-\frac{1}{\tan \alpha_{1}} \int_{R}\left\langle F_{0}\left(\mathbf{v}^{0}\right), \mathbf{v}_{\xi}^{0}\right\rangle \mathrm{d} \xi \mathrm{d} \eta .
\end{aligned}
$$

Since $\mathbf{v}^{0}$ satisfies (4.5), it holds by integration by parts that

$$
\begin{aligned}
& \int_{R}\left\langle F_{0}\left(\mathbf{v}^{0}\right), \mathbf{v}_{\xi}^{0}\right\rangle \mathrm{d} \xi \mathrm{d} \eta \\
& =-\int_{R}\left\langle D \Delta \mathbf{v}^{0}, \mathbf{v}_{\xi}^{0}\right\rangle \mathrm{d} \xi \mathrm{d} \eta \\
& =-\int_{\partial R}\left\langle D \Delta \mathbf{v}^{0}, \mathbf{v}^{0}\right\rangle \nu^{\xi} \mathrm{d} s+\int_{R}\left\langle D \Delta \mathbf{v}_{\xi}^{0}, \mathbf{v}^{0}\right\rangle \mathrm{d} \xi \mathrm{d} \eta \\
& \simeq-\int_{\partial R}\left\langle D \Delta \mathbf{v}^{0}, \mathbf{v}^{0}\right\rangle \nu^{\xi} \mathrm{d} s+\int_{R}\left\langle\mathbf{v}_{\xi}^{0}, D \Delta \mathbf{v}^{0}\right\rangle \mathrm{d} \xi \mathrm{d} \eta,
\end{aligned}
$$

because (4.11) holds on $\partial R$. Hence, by (4.6) we have

$$
\begin{aligned}
\int_{R} & \left\langle F_{0}\left(\mathbf{v}^{0}\right), \mathbf{v}_{\xi}^{0}\right\rangle \mathrm{d} \xi \mathrm{d} \eta \\
& =-\int_{R}\left\langle D \Delta \mathbf{v}^{0}, \mathbf{v}_{\xi}^{0}\right\rangle \mathrm{d} \xi \mathrm{d} \eta \\
& =-\frac{1}{2} \int_{\partial R}\left\langle D \Delta \mathbf{v}^{0}, \mathbf{v}^{0}\right\rangle v^{\xi} \mathrm{d} s \\
& \simeq-\frac{1}{2} \int_{S_{1}}\left\langle D \Psi_{s s}^{1}, \Psi^{1}\right\rangle\left(-\sin \alpha_{3}\right) \mathrm{d} s-\frac{1}{2} \int_{S_{3}}\left\langle D \Psi_{s s}^{3}, \Psi^{3}\right\rangle \sin \alpha_{1} \mathrm{~d} s \\
& =\frac{1}{2}\left(M_{3} \sin \alpha_{1}-M_{1} \sin \alpha_{3}\right) .
\end{aligned}
$$

It follows from (4.19) and (4.21) that

$$
\begin{aligned}
K_{3} & \simeq-\frac{1}{\tan \alpha} \times \frac{1}{2}\left(M_{3} \sin \alpha_{1}-M_{1} \sin \alpha_{3}\right) \\
& =-\frac{1}{2} M_{3} \cos \alpha_{1}+\frac{1}{2} M_{1} \frac{\sin \alpha_{3}}{\tan \alpha_{1}} .
\end{aligned}
$$

Therefore, by (4.15), (4.16) and (4.17), (4.22), the quantity (4.14) is written as

$$
\iint_{R}\left(\int_{\Xi_{1}(\eta)}^{\xi}\left\langle F_{0}\left(\mathbf{v}^{0}\right), \mathbf{v}_{\xi}^{0}\right\rangle \mathrm{d} \xi^{\prime}\right)_{\eta} \mathrm{d} \xi \mathrm{d} \eta \simeq \frac{1}{2} M_{2}-\frac{1}{2} M_{3} \cos \alpha_{1}+\frac{1}{2} M_{1} \frac{\sin \alpha_{3}}{\tan \alpha_{1}} .
$$

On the other hand, the integrand of (4.14) is expanded into

$$
\begin{aligned}
& \left(\int_{\Xi_{1}(\eta)}^{\xi}\left\langle F_{0}\left(\mathbf{v}^{0}\right), \mathbf{v}_{\xi}^{0}\right\rangle \mathrm{d} \xi^{\prime}\right)_{\eta} \\
& =\int_{\Xi_{1}(\eta)}^{\xi}\left\{\left\langle F_{0}^{\prime}\left(\mathbf{v}^{0}\right) \mathbf{v}_{\eta}^{0}, \mathbf{v}_{\xi}^{0}\right\rangle+\left\langle F_{0}\left(\mathbf{v}^{0}\right), \mathbf{v}_{\xi \eta}^{0}\right\rangle\right\} \mathrm{d} \xi^{\prime}-\left.\frac{\mathrm{d} \Xi_{1}}{\mathrm{~d} \eta}\left\langle F_{0}\left(\mathbf{v}^{0}\right), \mathbf{v}_{\xi}^{0}\right\rangle\right|_{(\xi, \eta) \in S_{1}} .
\end{aligned}
$$


Here, $\frac{\mathrm{d} \Xi_{1}}{\mathrm{~d} \eta}=-\frac{1}{\tan \alpha_{3}}, \mathbf{v}^{0} \simeq \Psi^{1}\left(\xi_{1}\right)$ and $\mathbf{v}_{\xi}^{0} \simeq \Psi_{s}^{1} \cos \alpha_{3}$ hold on the side $S_{1}$, which imply

$$
-\left.\frac{\mathrm{d} \Xi_{1}}{\mathrm{~d} \eta}\left\langle F_{0}\left(\mathbf{v}^{0}\right), \mathbf{v}_{\xi}^{0}\right\rangle\right|_{S_{1}}=\frac{\cos ^{2} \alpha_{3}}{\sin \alpha_{3}}\left\langle F_{0}\left(\Psi^{1}\right), \Psi_{s}^{1}\right\rangle .
$$

Since $\mathbf{v}_{\eta}^{0} \simeq \Psi_{s}^{1} \times\left(-\sin \alpha_{3}\right)$ on the side $S_{1}$, it follows

$$
\begin{aligned}
& \int_{\Xi_{1}(\eta)}^{\xi}\left\langle F_{0}\left(\mathbf{v}^{0}\right), \mathbf{v}_{\xi \eta}^{0}\right\rangle \mathrm{d} \xi^{\prime} \\
& =\left[\left\langle F_{0}\left(\mathbf{v}^{0}\right), \mathbf{v}_{\eta}^{0}\right\rangle\right]_{\Xi_{1}(\eta)}^{\xi}-\int_{\Xi_{1}(\eta)}^{\xi}\left\langle F_{0}^{\prime}\left(\mathbf{v}^{0}\right) \mathbf{v}_{\xi}^{0}, \mathbf{v}_{\eta}^{0}\right\rangle \mathrm{d} \xi^{\prime} \\
& =\left\langle F_{0}\left(\mathbf{v}^{0}\right), \mathbf{v}_{\eta}^{0}\right\rangle-\left\langle F_{0}\left(\Psi^{1}\right), \Psi_{s}^{1}\right\rangle\left(-\sin \alpha_{3}\right)-\int_{\Xi_{1}(\eta)}^{\xi}\left\langle F_{0}^{\prime}\left(\mathbf{v}^{0}\right) \mathbf{v}_{\xi}^{0}, \mathbf{v}_{\eta}^{0}\right\rangle \mathrm{d} \xi^{\prime} .
\end{aligned}
$$

From (4.25) and (4.26), Eqn (4.24) becomes

$$
\begin{aligned}
& \left(\int_{\Xi_{1}(\eta)}^{\xi}\left\langle F_{0}\left(\mathbf{v}^{0}\right), \mathbf{v}_{\xi}^{0}\right\rangle \mathrm{d} \xi^{\prime}\right)_{\eta} \\
& =\int_{\Xi_{1}(\eta)}^{\xi}\left\{\left\langle F_{0}^{\prime}\left(\mathbf{v}^{0}\right) \mathbf{v}_{\eta}^{0}, \mathbf{v}_{\xi}^{0}\right\rangle-\left\langle F_{0}^{\prime}\left(\mathbf{v}^{0}\right) \mathbf{v}_{\xi}^{0}, \mathbf{v}_{\eta}^{0}\right\rangle\right\} \mathrm{d} \xi^{\prime} \\
& +\left\langle F_{0}\left(\mathbf{v}^{0}\right), \mathbf{v}_{\eta}^{0}\right\rangle+\left(\sin \alpha_{3}+\frac{\cos ^{2} \alpha_{3}}{\sin \alpha_{3}}\right)\left\langle F_{0}\left(\Psi^{1}\right), \Psi_{s}^{1}\right\rangle \\
& =\int_{\Xi_{1}(\eta)}^{\xi}\left\{\left\langle F_{0}^{\prime}\left(\mathbf{v}^{0}\right) \mathbf{v}_{\eta}^{0}, \mathbf{v}_{\xi}^{0}\right\rangle-\left\langle F_{0}^{\prime}\left(\mathbf{v}^{0}\right) \mathbf{v}_{\xi}^{0}, \mathbf{v}_{\eta}^{0}\right\rangle\right\} \mathrm{d} \xi^{\prime} \\
& +\left\langle F_{0}\left(\mathbf{v}^{0}\right), \mathbf{v}_{\eta}^{0}\right\rangle+\frac{1}{\sin \alpha_{3}}\left\langle F_{0}\left(\Psi^{1}\right), \Psi_{s}^{1}\right\rangle .
\end{aligned}
$$

Let $\eta=H(\xi)$ be the value of $\eta$-coordinate of a point $(\xi, \eta) \in S_{1} \cup S_{3}$, that is,

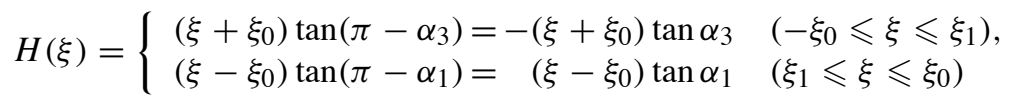

and $R(\xi)$ be a subdomain of $R$ given by

$$
R(\xi)=\left\{\left(\xi^{\prime}, \eta\right) \in R ;-\xi_{0} \leqslant \xi^{\prime} \leqslant \xi\right\} .
$$

Then, the integration of (4.27) over $R$ becomes

$$
\begin{gathered}
\iint_{R}\left(\int_{\Xi_{1}(\eta)}^{\xi}\left\langle F_{0}\left(\mathbf{v}^{0}\right), \mathbf{v}_{\xi}^{0}\right\rangle \mathrm{d} \xi^{\prime}\right)_{\eta} \mathrm{d} \xi \mathrm{d} \eta \\
=\int_{-\xi_{0}}^{\xi_{0}} \int_{-\eta_{0}}^{H(\xi)} \int_{\Xi_{1}(\eta)}^{\xi}\left\{\left\langle F_{0}^{\prime}\left(\mathbf{v}^{0}\right) \mathbf{v}_{\eta}^{0}, \mathbf{v}_{\xi}^{0}\right\rangle-\left\langle F_{0}^{\prime}\left(\mathbf{v}^{0}\right) \mathbf{v}_{\xi}^{0}, \mathbf{v}_{\eta}^{0}\right\rangle\right\} \mathrm{d} \xi^{\prime} \mathrm{d} \eta \mathrm{d} \xi
\end{gathered}
$$




$$
\begin{gathered}
+\iint_{R}\left\langle F_{0}\left(\mathbf{v}^{0}\right), \mathbf{v}_{\eta}^{0}\right\rangle \mathrm{d} \xi \mathrm{d} \eta+\frac{1}{\sin \alpha_{3}} \int_{-\eta_{0}}^{\eta_{1}} \int_{\Xi_{1}(\eta)}^{\Xi_{3}(\eta)}\left\langle F_{0}\left(\Psi^{1}\right), \Psi_{s}^{1}\right\rangle \mathrm{d} \xi \mathrm{d} \eta \\
=\int_{-\xi_{0}}^{\xi_{0}}\left(\iint_{R(\xi)}\left\{\left\langle F_{0}^{\prime}\left(\mathbf{v}^{0}\right) \mathbf{v}_{\eta}^{0}, \mathbf{v}_{\xi}^{0}\right\rangle-\left\langle F_{0}^{\prime}\left(\mathbf{v}^{0}\right) \mathbf{v}_{\xi}^{0}, \mathbf{v}_{\eta}^{0}\right\rangle\right\} \mathrm{d} \xi^{\prime} \mathrm{d} \eta\right) \mathrm{d} \xi \\
\quad+\iint_{R}\left\langle F_{0}\left(\mathbf{v}^{0}\right), \mathbf{v}_{\eta}^{0}\right\rangle \mathrm{d} \xi \mathrm{d} \eta+\frac{1}{\sin \alpha_{3}} \int_{-\eta_{0}}^{\eta_{1}}\left(\Xi_{3}(\eta)-\Xi_{1}(\eta)\right)\left\langle F_{0}\left(\Psi^{1}\right), \Psi_{s}^{1}\right\rangle \mathrm{d} \eta \\
=K_{1}^{\prime}+K_{2}^{\prime}+K_{3}^{\prime} .
\end{gathered}
$$

Now, we compute each term $K_{i}^{\prime}(i=1,2,3)$ in the last equation of (4.28). First, we know

$$
\begin{aligned}
K_{2}^{\prime} & =\iint_{R}\left\langle F_{0}\left(\mathbf{v}^{0}\right), \mathbf{v}_{\eta}^{0}\right\rangle \mathrm{d} \xi \mathrm{d} \eta \\
& =-\iint_{R}\left\langle D \Delta \mathbf{v}^{0}, \mathbf{v}_{\eta}^{0}\right\rangle \mathrm{d} \xi \mathrm{d} \eta \\
& =-\int_{\partial R}\left\langle D \Delta \mathbf{v}^{0}, \mathbf{v}^{0}\right\rangle v^{\eta} \mathrm{d} s+\iint_{R}\left\langle D \Delta \mathbf{v}_{\eta}^{0}, \mathbf{v}^{0}\right\rangle \mathrm{d} \xi \mathrm{d} \eta \\
& \simeq-\int_{\partial R}\left\langle D \Delta \mathbf{v}^{0}, \mathbf{v}^{0}\right\rangle v^{\eta} \mathrm{d} s+\iint_{R}\left\langle\mathbf{v}_{\eta}^{0}, D \Delta \mathbf{v}^{0}\right\rangle \mathrm{d} \xi \mathrm{d} \eta,
\end{aligned}
$$

because (4.11) holds on the boundary $\partial R$. Hence, we obtain

$$
\begin{aligned}
K_{2}^{\prime} & =-\iint_{R}\left\langle D \Delta \mathbf{v}^{0}, \mathbf{v}_{\eta}^{0}\right\rangle \mathrm{d} \xi \mathrm{d} \eta \\
\simeq & -\frac{1}{2} \int_{\partial R}\left\langle D \Delta \mathbf{v}^{0}, \mathbf{v}^{0}\right\rangle v^{\eta} \mathrm{d} s \\
\simeq & -\frac{1}{2}\left\{\int_{S_{2}}\left\langle D \Psi_{s s}^{2}, \Psi^{2}\right\rangle(-1) \mathrm{d} s+\int_{S_{3}}\left\langle D \Psi_{s s}^{3}, \Psi^{3}\right\rangle\left(-\cos \alpha_{1}\right) \mathrm{d} s\right. \\
& \left.\quad+\int_{S_{1}}\left\langle D \Psi_{s s}^{1}, \Psi^{1}\right\rangle\left(-\cos \alpha_{3}\right) \mathrm{d} s\right\} \\
& =-\frac{1}{2}\left(M_{2}+M_{3} \cos \alpha_{1}+M_{1} \cos \alpha_{3}\right) .
\end{aligned}
$$

Next, we consider $K_{3}^{\prime}=\frac{1}{\sin \alpha_{3}} \int_{-\eta_{0}}^{\eta_{1}}\left(\Xi_{3}(\eta)-\Xi_{1}(\eta)\right)\left\langle F_{0}\left(\Psi^{1}\right), \Psi_{s}^{1}\right\rangle \mathrm{d} \eta$. Let

$$
\begin{aligned}
\Xi(\eta) & =\Xi_{3}(\eta)-\Xi_{1}(\eta) \\
& =\left(\frac{1}{\tan \alpha_{1}}+\frac{1}{\tan \alpha_{3}}\right) \eta+Q,
\end{aligned}
$$

where $Q$ is a constant given by

$$
Q=2 \xi_{0}+\left(\frac{1}{\tan \alpha_{1}}+\frac{1}{\tan \alpha_{3}}\right) \eta_{0} .
$$


Note that $\mathrm{d} \eta=-\sin \alpha_{3} \mathrm{~d} s$ and

$$
\frac{\mathrm{d} \Xi}{\mathrm{d} s}=-\sin \alpha_{3} \frac{\mathrm{d} \Xi}{\mathrm{d} \eta}=-\sin \alpha_{3} \times\left(\frac{1}{\tan \alpha_{1}}+\frac{1}{\tan \alpha_{3}}\right)
$$

hold on the side $S_{1}$. Then, by (4.12) and (4.13), we know

$$
\begin{aligned}
K_{3}^{\prime} & =\frac{1}{\sin \alpha_{3}} \int_{S_{1}} \Xi(\eta)\left\langle F\left(\Psi^{1}\right), \Psi_{s}^{1}\right\rangle\left(-\sin \alpha_{3}\right) \mathrm{d} s \\
& \simeq \int_{-\infty}^{\infty} \Xi(\eta)\left\langle F\left(\Psi^{1}\right), \Psi_{s}^{1}\right\rangle \mathrm{d} s \\
& =\left[\Xi(\eta) \int_{-\infty}^{s}\left\langle F\left(\Psi^{1}\right), \Psi_{s}^{1}\right\rangle \mathrm{d} s^{\prime}\right]_{-\infty}^{\infty} \\
& =\left[\Xi \times\left(-\frac{1}{2}\right)\left\langle D \Psi_{s}^{1}, \Psi_{s}^{1}\right\rangle\right]_{-\infty}^{\infty}-\frac{1}{2} \sin \alpha_{3}\left(\frac{1}{\tan \alpha_{1}}+\frac{1}{\tan \alpha_{3}}\right) M_{1}^{\infty} \int_{-\infty}^{s}\left\langle F\left(\Psi^{1}\right), \Psi_{s}^{1}\right\rangle \mathrm{d} s^{\prime} \mathrm{d} s \\
& =-\frac{1}{2} \sin \alpha_{3}\left(\frac{1}{\tan \alpha_{1}}+\frac{1}{\tan \alpha_{3}}\right) M_{1}
\end{aligned}
$$

Finally, we compute $K_{1}^{\prime}$. Differentiating (4.5) with respect to $\xi$ or $\eta$, we obtain

$$
\left\{\begin{array}{l}
F_{0}^{\prime}\left(\mathbf{v}^{0}\right) \mathbf{v}_{\xi}^{0}=-D \Delta \mathbf{v}_{\xi}^{0} \\
F_{0}^{\prime}\left(\mathbf{v}^{0}\right) \mathbf{v}_{\eta}^{0}=-D \Delta \mathbf{v}_{\eta}^{0}
\end{array}\right.
$$

Let $l(\xi)$ be the line segment defined by

$$
l(\xi)=\left\{(\xi, \eta) ;-\eta_{0} \leqslant \eta \leqslant H(\xi)\right\}
$$

Noting (4.11) holds on the sides $S_{1} \cup S_{3}$, and using (4.31), we know

$$
\begin{aligned}
& \iint_{R(\xi)}\left\langle F_{0}^{\prime}\left(\mathbf{v}^{0}\right) \mathbf{v}_{\eta}^{0}, \mathbf{v}_{\xi}^{0}\right\rangle \mathrm{d} \xi^{\prime} \mathrm{d} \eta \\
& \quad=-\iint_{R(\xi)}\left\langle D \Delta \mathbf{v}_{\eta}^{0}, \mathbf{v}_{\xi}^{0}\right\rangle \mathrm{d} \xi^{\prime} \mathrm{d} \eta \\
& \quad \simeq-\iint_{R(\xi)}\left\langle\mathbf{v}_{\eta}^{0}, D \Delta \mathbf{v}_{\xi}^{0}\right\rangle-\int_{l(\xi)}\left\{\left\langle D \frac{\partial \mathbf{v}_{\eta}^{0}}{\partial v}, \mathbf{v}_{\xi}^{0}\right\rangle-\left\langle D \frac{\partial \mathbf{v}_{\xi}^{0}}{\partial v}, \mathbf{v}_{\eta}^{0}\right\rangle\right\} \mathrm{d} s \\
& \quad=-\iint_{R(\xi)}\left\langle\mathbf{v}_{\eta}^{0}, D \Delta \mathbf{v}_{\xi}^{0}\right\rangle-\int_{-\eta_{0}}^{H(\xi)}\left\{\left\langle D \mathbf{v}_{\eta \xi}^{0}, \mathbf{v}_{\xi}^{0}\right\rangle-\left\langle D \mathbf{v}_{\xi \xi}^{0}, \mathbf{v}_{\eta}^{0}\right\rangle\right\} \mathrm{d} \eta \\
& \quad=\iint_{R(\xi)}\left\langle\mathbf{v}_{\eta}^{0}, F_{0}^{\prime}\left(\mathbf{v}^{0}\right) \mathbf{v}_{\xi}^{0}\right\rangle-\int_{-\eta_{0}}^{H(\xi)}\left\{\left\langle D \mathbf{v}_{\eta \xi}^{0}, \mathbf{v}_{\xi}^{0}\right\rangle-\left\langle D \mathbf{v}_{\xi \xi}^{0}, \mathbf{v}_{\eta}^{0}\right\rangle\right\} \mathrm{d} \eta
\end{aligned}
$$


where $v$ is the outward normal unit vector of $R(\xi)$ on the line segment $l(\xi)$. Here, we used the fact that the derivative in the direction of $v$ on $l(\xi)$ means the derivative with respect to $\xi$-variable. Thus, we find

$$
\begin{gathered}
\iint_{R(\xi)}\left\{\left\langle F_{0}^{\prime}\left(\mathbf{v}^{0}\right) \mathbf{v}_{\eta}^{0}, \mathbf{v}_{\xi}^{0}\right\rangle-\left\langle F_{0}^{\prime}\left(\mathbf{v}^{0}\right) \mathbf{v}_{\xi}^{0}, \mathbf{v}_{\eta}^{0}\right\rangle\right\} \mathrm{d} \xi^{\prime} \mathrm{d} \eta \\
=-\int_{-\eta_{0}}^{H(\xi)}\left\{\left\langle D \mathbf{v}_{\eta \xi}^{0}, \mathbf{v}_{\xi}^{0}\right\rangle-\left\langle D \mathbf{v}_{\xi \xi}^{0}, \mathbf{v}_{\eta}^{0}\right\rangle\right\} \mathrm{d} \eta .
\end{gathered}
$$

Now, we note that

$$
\mathbf{v}_{\xi}^{0} \simeq\left\{\begin{array}{lll}
\Psi_{s}^{1} \cos \alpha_{3}, & \text { on } & S_{1}, \\
\Psi_{s}^{3} \cos \alpha_{1}, & \text { on } & S_{3}
\end{array}\right.
$$

and

$$
\mathbf{v}_{\eta}^{0} \simeq\left\{\begin{array}{rll}
-\Psi_{s}^{1} \sin \alpha_{3}, & \text { on } & S_{1}, \\
\Psi_{s}^{3} \sin \alpha_{1}, & \text { on } & S_{3}
\end{array}\right.
$$

hold. Hence, by (4.33), $K_{1}^{\prime}$ is calculated as

$$
\begin{aligned}
& K_{1}^{\prime} \\
& \simeq-\int_{-\xi_{0}}^{\xi_{0}} \int_{-\eta_{0}}^{H(\xi)}\left\{\left\langle D \mathbf{v}_{\eta \xi}^{0}, \mathbf{v}_{\xi}^{0}\right\rangle-\left\langle D \mathbf{v}_{\xi \xi}^{0}, \mathbf{v}_{\eta}^{0}\right\rangle\right\} \mathrm{d} \eta \\
& =\int_{R}\left\{\left\langle D \mathbf{v}_{\xi \xi}^{0}, \mathbf{v}_{\eta}^{0}\right\rangle-\left\langle D \mathbf{v}_{\eta \xi}^{0}, \mathbf{v}_{\xi}^{0}\right\rangle\right\} \mathrm{d} \eta \mathrm{d} \xi \\
& =\int_{\partial R}\left\langle D \mathbf{v}_{\xi}^{0}, \mathbf{v}_{\eta}^{0}\right\rangle v^{\xi} \mathrm{d} s-2 \int_{R}\left\langle D \mathbf{v}_{\xi}^{0}, \mathbf{v}_{\xi \eta}^{0}\right\rangle \mathrm{d} \eta \mathrm{d} \xi \\
& \simeq \int_{S_{1}}\left\langle D \Psi_{s}^{1}, \Psi_{s}^{1}\right\rangle \cos \alpha_{3} \cdot\left(-\sin \alpha_{3}\right) \cdot\left(-\sin \alpha_{3}\right) \mathrm{d} s \\
& \quad+\int_{S_{3}}\left\langle D \Psi_{s}^{3}, \Psi_{s}^{3}\right\rangle \cos \alpha_{1} \cdot \sin \alpha_{1} \cdot \sin \alpha_{1} \mathrm{~d} s-\iint_{R} \frac{\partial}{\partial \eta}\left\langle D \mathbf{v}_{\xi}^{0}, \mathbf{v}_{\xi}^{0}\right\rangle \mathrm{d} \eta \mathrm{d} \xi \\
& \simeq M_{1} \sin ^{2} \alpha_{3} \cos \alpha_{3}+M_{3} \sin ^{2} \alpha_{1} \cos \alpha_{1}-\int_{\partial R}\left\langle D \mathbf{v}_{\xi}^{0}, \mathbf{v}_{\xi}^{0}\right\rangle v^{\eta} \mathrm{d} s .
\end{aligned}
$$

Here, $\int_{\partial R}\left\langle D \mathbf{v}_{\xi}^{0}, \mathbf{v}_{\xi}^{0}\right\rangle v^{\eta} \mathrm{d} s$ in the last term of (4.34) is written as

$$
\begin{aligned}
& \int_{\partial R}\left\langle D \mathbf{v}_{\xi}^{0}, \mathbf{v}_{\xi}^{0}\right\rangle v^{\eta} \mathrm{d} s \\
& \simeq \int_{S_{1}}\left\langle D \Psi_{s}^{1}, \Psi_{s}^{1}\right\rangle \cos ^{2} \alpha_{3} \cdot\left(-\cos \alpha_{3}\right) \mathrm{d} s+\int_{S_{2}}\left\langle D \Psi_{s}^{2}, \Psi_{s}^{2}\right\rangle(-1) \mathrm{d} s \\
& +\int_{S_{3}}\left\langle D \Psi_{s}^{3}, \Psi_{s}^{3}\right\rangle \cos ^{2} \alpha_{1} \cdot\left(-\cos \alpha_{1}\right) \mathrm{d} s \\
& =-M_{1} \cos ^{3} \alpha_{3}-M_{2}-M_{3} \cos ^{3} \alpha_{1} \text {. }
\end{aligned}
$$

Substituting the above into (4.34), we have

$$
K_{1}^{\prime} \simeq M_{1} \cos \alpha_{3}+M_{2}+M_{3} \cos \alpha_{1} .
$$


Thus, it follows from (4.30), (4.29) and (4.35) that

$$
\begin{aligned}
\iint_{R}\left(\int_{\Xi_{1}(\eta)}^{\xi}\left\langle F_{0}\left(\mathbf{v}^{0}\right), \mathbf{v}_{\xi}^{0}\right\rangle \mathrm{d} \xi^{\prime}\right)_{\eta} \mathrm{d} \xi \mathrm{d} \eta & \simeq K_{1}^{\prime}+K_{2}^{\prime}+K_{3}^{\prime} \\
& =-\frac{1}{2} M_{1} \frac{\sin \alpha_{3}}{\tan \alpha_{1}}+\frac{1}{2} M_{2}+\frac{1}{2} M_{3} \cos \alpha_{1}
\end{aligned}
$$

(4.23) and (4.36) imply

$$
\frac{M_{1}}{\sin \alpha_{1}} \simeq \frac{M_{3}}{\sin \alpha_{3}}
$$

and similarly, $\frac{M_{1}}{\sin \alpha_{1}} \simeq \frac{M_{2}}{\sin \alpha_{2}}$ holds. We thus arrive at the following angle condition at the triple junction point $\gamma$ :

$$
\frac{M_{1}}{\sin \alpha_{1}}=\frac{M_{2}}{\sin \alpha_{2}}=\frac{M_{3}}{\sin \alpha_{3}}
$$

by taking $\triangle A B C$ infinitely large.

It should be emphasized that our system is a non-gradient system but the formula (4.38) is exactly the same as the one derived from gradient systems with triple well potential [1]. Thus, the limiting problem to describe interfaces with triple junctions can be derived from the RD system (1.8), by using (4.1) and (4.38). The local existence result of this problem is shown in [1].

There are some numerical methods which have been developed to track motions of triple junctions. We should refer to $[2,17]$, for example.

Although the derivation of (4.38) has been carried out by means of formal asymptotic analysis, we could numerically confirm that the dynamics of interfaces (2.5) supplemented with the angle condition (4.38) in time-scale $T=\epsilon^{2} t$ is a good approximation to that of the internal layers of the RD system (1.8) when $\epsilon$ is sufficiently small, as in Fig. 8.

\section{Concluding remarks}

We have obtained the angle condition (4.38) at each triple junction, assuming that $\theta_{1}, \theta_{2}$ and $\theta_{3}$ are all sufficiently small.

First we consider the motion of interfacial curves (2.5). A closed curve in $\mathbf{R}^{2}$ exhibit the curve shortening property when its dynamics is governed by mean curvature flow [5]. It is also indicated that the same property holds in the case of interfaces with triple junctions if all the angles are equal and velocities $\theta_{1}, \theta_{2}$ and $\theta_{3}$ vanish [18]. This fact shows that surface energy decreases with time, but the angle condition (4.38) guarantees that the interfacial energy preserves at triple junctions; we define the energy $E$ as

$$
E=M_{1} l_{1}+M_{2} l_{2}+M_{3} l_{3},
$$

where $l_{i}$ is the length of $\Gamma_{i}(i=1,2,3)$. Changing rate of $E$ at the junction point is

$$
\begin{aligned}
-M_{1}\left(\gamma_{T} \cdot v_{1}\right)-M_{2}\left(\gamma_{T} \cdot v_{2}\right)-M_{3}\left(\gamma_{T} \cdot v_{3}\right) & =-\gamma_{T} \cdot\left(M_{1} v_{1}+M_{2} v_{2}+M_{3} v_{3}\right) \\
& =-\gamma_{T} \cdot 0 \\
& =0 .
\end{aligned}
$$



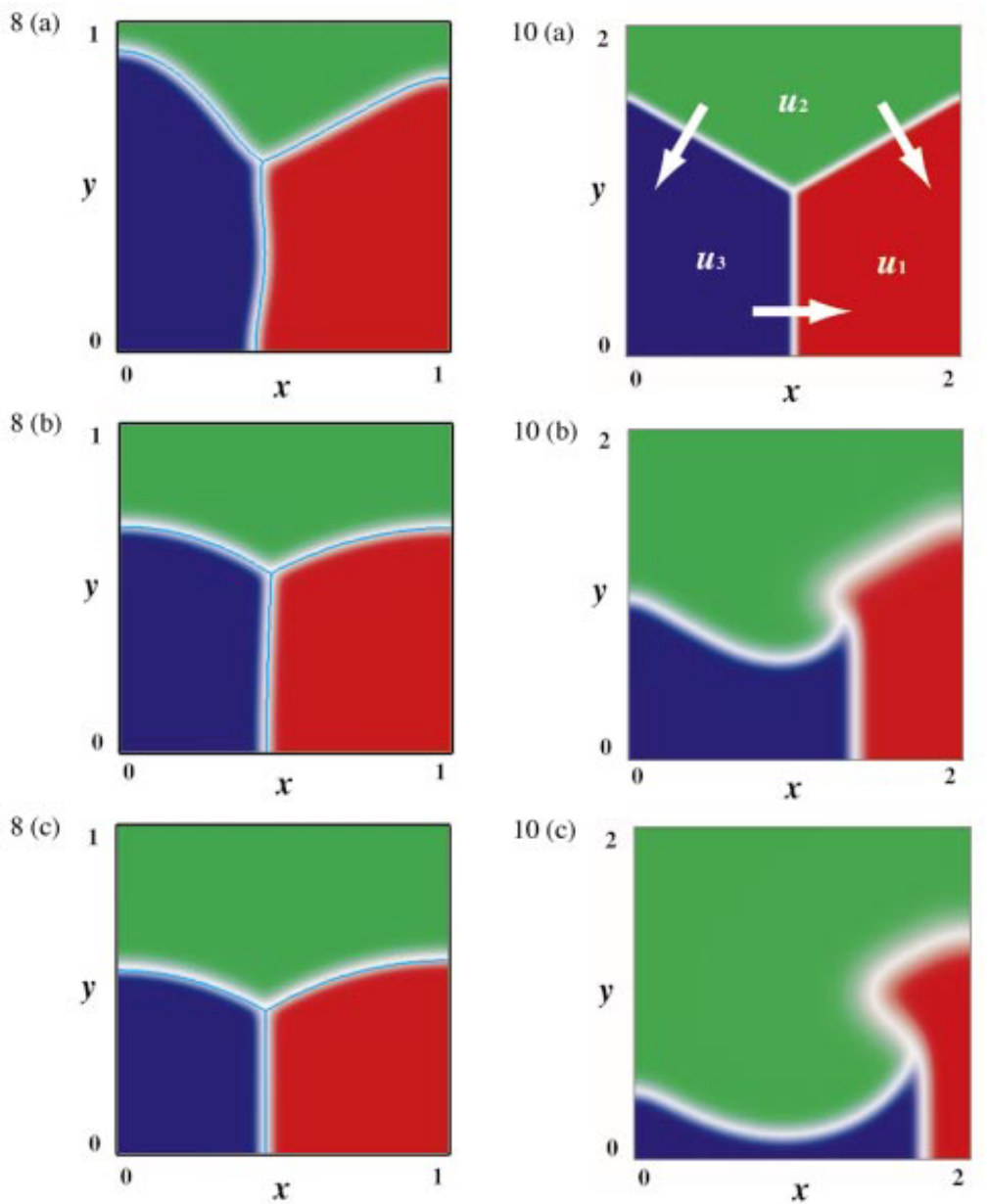

FIG. 8. Comparison of phase-separating pattern of the RD system (1.8) and interfaces with triple juction as $\epsilon \downarrow 0$. The parameters are completely the same as those in Fig. 1. Here $M_{i}=0.33795$. (a) $t=0(T=0.0)$, (b) $t=1000(T=0.1)$, (c) $t=2000(T=0.2)$.

FIG. 10. Dynamics of interfaces with triple junctions in (1.8)-(1.10), where $\epsilon=1.0 \times 10^{-2}, d_{i}=11 / 6 d_{2}=4$, $d_{3}=1, r_{1}=1 / 8 r_{2}=1, r_{3}=1$ and

$$
\left(\begin{array}{lll}
a_{11} & a_{12} & a_{13} \\
a_{21} & a_{22} & a_{23} \\
a_{31} & a_{32} & a_{33}
\end{array}\right)=\left(\begin{array}{ccc}
1 / 18 & 11 / 72 & 11 / 18 \\
13 / 8 & 1 & 4 / 3 \\
7 / 6 & 7 / 3 & 1
\end{array}\right) .
$$

(a) $t=0$, (b) $t=60$, (c) $t=120$.

For these parameters, $\theta_{1}=\frac{2}{\sqrt{6}}, \theta_{2}=\frac{3}{2 \sqrt{6}}$ and $\theta_{3}=-\frac{1}{4 \sqrt{6}}$. 


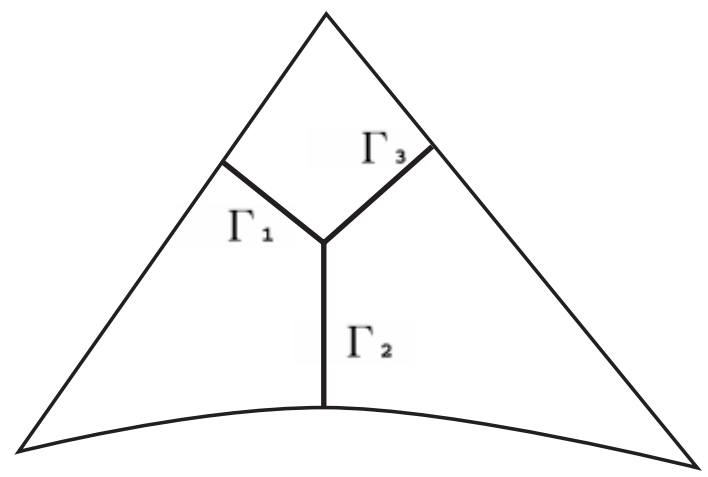

FIG. 9. Stationary interfaces with a triple junction in some triangular domain.

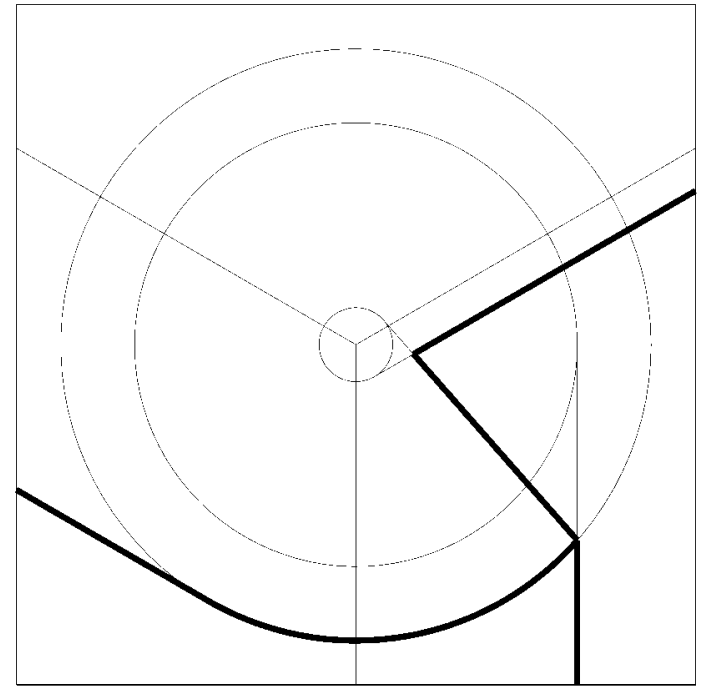

FIG. 11. Taylor's motion of interfaces. 
On the other hand, Lemma 3.1.2 in [5] is modified for an open curve as follows;

$$
\frac{\mathrm{d} l_{i}(t)}{\mathrm{d} t}=-L_{i} \int_{\Gamma_{i}} \kappa_{i}^{2} \mathrm{~d} s+\left[\frac{\partial \Gamma_{i}}{\partial t} \cdot \frac{\partial \Gamma_{i}}{\partial s}\right]_{0}^{l_{i}},
$$

where $s$ is the arc length parameter. Thus we get

$$
\begin{aligned}
\frac{\mathrm{d} E}{\mathrm{~d} t}= & -M_{1}\left(L_{1} \int_{\Gamma_{1}} \kappa_{1}^{2} \mathrm{~d} s-\left(-\gamma_{T} \cdot v_{1}\right)\right)-M_{2}\left(L_{2} \int_{\Gamma_{2}} \kappa_{2}^{2} \mathrm{~d} s-\left(-\gamma_{T} \cdot v_{2}\right)\right) \\
& -M_{3}\left(L_{3} \int_{\Gamma_{3}} \kappa_{3}^{2} \mathrm{~d} s-\left(-\gamma_{T} \cdot \nu_{3}\right)\right) \\
= & -M_{1} L_{1} \int_{\Gamma_{1}} \kappa_{1}^{2} \mathrm{~d} s-M_{2} L_{2} \int_{\Gamma_{2}} \kappa_{2}^{2} \mathrm{~d} s-M_{3} L_{3} \int_{\Gamma_{3}} \kappa_{3}^{2} \mathrm{~d} s \\
\leqslant & 0 .
\end{aligned}
$$

From this result, we can conjecture that a stationary solution will be stable, if $\Omega$ is specified as some suitable triangular domain as in Fig. 9. More general results on properties of energy are obtained in [16].

Secondly we consider the RD system (1.8) when parameters are fixed such that the velocities $\theta_{1}, \theta_{2}$ and $\theta_{3}$ satisfy $\theta_{1}=\frac{2}{\sqrt{6}}, \theta_{2}=\frac{3}{2 \sqrt{6}}$ and $\theta_{3}=-\frac{1}{4 \sqrt{6}}$ [15]. The resulting numerical pattern qualitatively resembles the unique consistent motion constructed by Taylor [19]. However, behavior of interfaces arising in Figs 4 and 5 cannot be understood through the notion of the unique consistent motion. As far as we know, there is no theory which explains why such spirals stably exist. According to [19], if $0<\theta_{1}<\theta_{2}<\theta_{3}$, then a triple junction point moves with a constant velocity in one direction. The results of numerical simulations cannot be understood from this point of view. We must, therefore, develop another approach to this problem.

\section{REFERENCES}

1. Bronsard, L. \& ReITICH, F. On three-phase boundary motion and the singular limit of a vector-valued Ginzburg-Landau equation. Arch. Rat. Mech. 124, (1993) 355-379.

2. Bronsard, L. \& Wetton, B. T. R. A Numerical method for tracking curve networks moving with curvature motion. J. Comput. Phys. 120, (1995) 66-87.

3. Dancer, E. N., Hilhorst, D., Mimura, M., \& Peletier, L. A. Spatial segregation limit of a competition-diffusion system. European J. Appl. Math. to appear.

4. EI, S.-I. \& YANAGIDA, E. Dynamics of interfaces in competition-diffusion systems. SIAM J. Appl. Math. 54, (1994) 1355-1373.

5. Gage, M. \& Hamilton, R. S. The heat equation shrinking convex plane curves. J. Differential Geom. 23, (1986) 69-96.

6. Gause, G. F. The Struggle for Existence. The Williams \& Wilkins Company, Baltimore (1934).

7. Hirsch, M. W. Differential equations and convergence almost everywhere of strongly monotone semiflows. PAM Technical Report, University of California, Berkeley (1982).

8. KAN-ON, Y. Parameter dependence of propagation speed of travelling waves for competition-diffusion equations. SIAM J. Math. Anal. 26, (1995) 340-363.

9. KAN-ON, Y. \& Mimura, M. Predation-mediated coexistence and segregation structures. Stud. Math. Appl. 18, (1986) 129-155. 
10. KAN-ON, Y. \& MimURA, M. Singular perturbation approach to a 3-component reaction-diffusion system arising in population dynamics. SIAM J. Math. Anal. 29, (1998) 1519-1536.

11. Kishimoto, K. \& Weinberger, H. F. The spatial homogeneity of stable equilibria of some reactiondiffusion systems on convex domains, J. Differential Equations 58, (1985) 15-21.

12. Matano, H. \& Mimura, M. Pattern formation in competition-diffusion systems in nonconvex domains. Publ. RIMS, Kyoto Univ. 19, (1983) 1049-1079.

13. MaY, R. M. \& LeOnARD, W. J. Nonlinear aspects of competition between three species. SIAM J. Appl. Math. 29, (1975) 243-253.

14. Mimura, M. \& Fife, P. C. A 3-component system of competition and diffusion. Hiroshima Math. J. 16, (1986) 189-207.

15. Mimura, M. \& Rodrigo, M. Exact travelling wave solutions of a competition-diffusion system, manuscript.

16. Novick-Cohen, A. Triple junction motion for Allen-Cahn/Cahn-Hilliard systems. Preprint (1997).

17. RuUth, S. J. A diffusion-generated approach to multiphase motion. J. Comput. Phys. 145, (1998) 166192.

18. Sternberg, P. \& Ziemer, W. P. Local minimizers of a three phase partition problem with triple junctions. Proc. Royal Soc. Edin. 124A, (1994) 1059-1073.

19. TAYLOR, J. E. The motion of multiple-phase junctions under prescribed phase-boundary velocities. $J$. Differential Equations 119, (1995) 109-136.

20. VAn Den Driessche, P. \& Zeeman, M. L. Three-dimensional competitive Lotka-Volterra systems with no periodic orbits. SIAM J. Appl. Math. 58, (1998) 227-234. 